\title{
Optimal Anti-Jam Attitude Determination using the Global Positioning System
}

\author{
Matthew Markel, Sverdrup Technology, Eric Sutton and Henry Zmuda, University of Florida
}

\begin{abstract}
This paper develops the mathematical basis for a GPS based attitude determination (AD) system that performs even in the presence of strong external interference. This is accomplished by approaching the AD problem from an antenna array viewpoint and using phase and amplitude information, vice using only phase information as in "conventional" AD systems. By redefining the array response vector used in array processing, a Maximum Likelihood Attitude Estimator (MLAE) is developed. This paper shows that the MLAE is a consistent estimator, and its performance asymptotically achieves the CramérRao Bound. Simulation based performance results of the MLAE are presented for various antenna topologies and interference scenarios, showing that it offers a significant improvement over the conventional attitude determination methods.
\end{abstract}

\section{INTRODUCTION}

It is well known that adaptive antenna arrays (often referred to as "Smart Antennas" for communications systems) provide significant resistance to unintentional interference and intentional jamming for both signal extraction and direction finding. Global Positioning System (GPS) based attitude determination (AD) systems utilize multiple sensors as well to extract attitude through carrier phase differences between the sensors [1]. However, present $\mathrm{AD}$ algorithms typically offer only the jamming resistance inherent in the receiver (i.e. gain from the spread spectrum waveform and perhaps coupling with some form of INS), which for even low powered jammers may not be enough to prevent corruption of the attitude estimates. Therefore, it seems appealing to exploit the similarities between the two fields of adaptive array processing and GPS based attitude determination to increase the anti-jam capabilities of GPS attitude systems. Indeed, this work centers around a substantially different method of approaching the attitude determination task than is typically employed.

\section{Conventional Phase Difference Model}

Consider the signal model and assumptions of a conventional attitude determination system. After downconversion, despreading, and integration the signal received at sensor $x_{0}$ of Figure 1 consists of a contribution from the satellite and a noise term.

$$
x_{0}=g e^{j \phi_{0}}+n_{0}
$$

The second sensor also receives the same satellite signal only shifted in time (i.e. phase).

$$
x_{1}=g e^{j\left(\phi_{0}+\delta \phi\right)}+n_{1}
$$

The noise terms $n_{0}$ and $n_{1}$ are assumed uncorrelated, and moreover are assumed to be small compared to the received gain from the satellite signal $g$ (after processing described above). The attitude determination problem

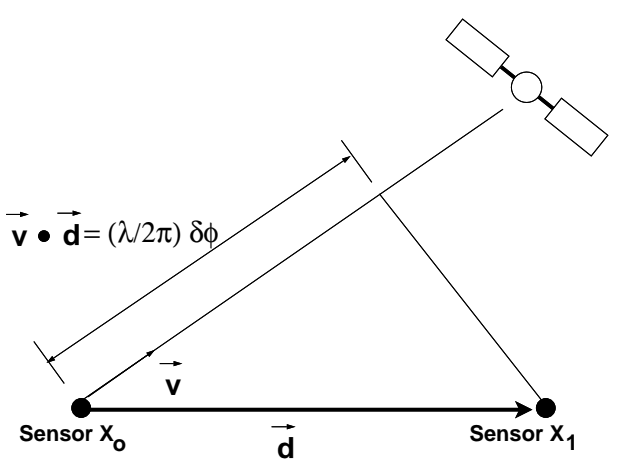

Fig. 1. A two sensor interferometric system. The baseline vector vector $\vec{d}$ can be determined by the known LOS vector $\vec{v}$ and the phase difference between the signals measured at the two sensors $x_{0}$ and $x_{1}$.

for this simple two sensor case ${ }^{1}$ is to estimate the baseline vector $\vec{d}$. It is clear to see that by taking the phase difference $\Delta \phi$ between $x_{0}$ and $x_{1}$, then the following relationship between the orientation of the vector $\vec{d}$, the known line of sight (LOS) vector to the satellite $\vec{v}$, and the phase difference may be obtained as [3]

$$
\begin{aligned}
\Delta \phi & =\Phi\left(x_{1}\right)-\Phi\left(x_{0}\right) \\
& \approx \frac{2 \pi}{\lambda}(\vec{v} \bullet \vec{d}+k \lambda) \\
& \approx \delta \phi+k(2 \pi)
\end{aligned}
$$

where $\Phi(\cdot)$ implies taking the phase angle of $(\cdot), \approx$ implies "is approximately equal to," and $k$ is an integer.

The reason that (5) holds is due to the previous assumption that the amplitude of the interference terms is much smaller than the amplitude of the satellite terms, and therefore the satellite components dominate the phase

\footnotetext{
${ }^{1}$ By using at least three sensors and two satellites [2], the complete attitude of the antenna may be found.
} 
of the sumof satellite signal and noise. However, when an interference source such as a jammer is present, the accuracy quickly degrades. This occurs because the presence of the jammer violates the signal model in that the phase difference, $\Delta \phi$, is no longer a good approximation of $\delta \phi$. Even with relatively high signal to jammer ratios, the performance of this conventional attitude estimator will be degraded. The fundamental thrust of this paper is the development of algorithms that increase the resistance of the attitude estimator to external interference.

The algorithms developed in this paper require that the satellites used for attitude determination are in code and Doppler track, even in the presence of jammers. This may be accomplished by using the receiver design of [4], where the data from multiple sensors are adaptively combined before they are used for updating the tracking loops. The adaptive weights are designed to provide gain towards the satellites, while providing attenuation in the direction of the jammers and therefore allowing successful tracking of the satellites.

\section{Notation, Preliminaries, and Signal Model}

We begin this section by reviewing the matrix and vector notation used throughout this work.

$$
\begin{aligned}
& \mathbf{A}^{T}=\text { the transpose of the matrix } \mathbf{A} \\
& \mathbf{A}^{H}=\text { the conjugate transpose of } \mathbf{A}
\end{aligned}
$$

$\mathbf{A} \odot \mathbf{B}=$ the Hadamard product of $\mathbf{A}$ and $\mathbf{B}$

$\mathbf{I}_{j}=$ the $j \times j$ identity matrix

$\mathbf{A}>0=\mathbf{A}$ is positive definite

$\mathbf{Z} \in \mathcal{C}^{a \times b}=\mathbf{Z}$ is a complex matrix of size $a \times b$

$\mathbf{q}_{1} \star \mathbf{q}_{2}=$ quaternion multiplication

In addition, the following variables are consistently used in the following roles.

$$
\begin{aligned}
L & =\text { the number of satellites being tracked } \\
M & =\text { the number of sensors in the array } \\
N & =\text { the number of snapshots of data available }
\end{aligned}
$$

Some common parameterizations of the attitude include Euler angles, quaternions, and direction cosine matrices. Throughout this work, this attitude is referred to generically as $q$, to remain independent of any particular parameterization. However, when specific parameterizations of this attitude are employed they follow the notation and description below.

$$
\begin{aligned}
q & =\text { a hypothesized attitude of the antenna } \\
o & =\text { the true attitude of the antenna } \\
q & =\left[q_{1}, q_{2}, q_{3}, q_{4}\right]^{T}, \text { the attitude quaternion } \\
\mathbf{q} & =\text { the } 3 \times 3 \text { direction cosine matrix }
\end{aligned}
$$

A common model for a snapshot of data, $\mathbf{x}\left(t_{n}\right) n=$ $1,2, \ldots, N$, received at the $M$ sensors

$$
\mathbf{x}\left(t_{n}\right)=\sum_{l=1}^{L} \gamma_{l} \mathbf{a}\left(\theta_{l}\right) s_{l}\left(t_{n}\right)+\mathbf{n}\left(t_{n}\right)
$$

where $\mathbf{n}(t), \mathbf{a}\left(\theta_{l}\right)$ and $\mathbf{x}(t) \in \mathcal{C}^{M \times 1}$. In equation (6), $\gamma_{l}$ and $\theta_{l}$ represent the unknown complex gain and direction corresponding to the $l$ th satellite source. The array response vector (spatial steering vector), $\mathbf{a}\left(\theta_{l}\right)$, is defined as the response of the array to a signal impinging from direction $\theta_{l}$, and is assumed known and unique for any possible $\theta_{l}$. The direction $\theta_{l}$, which is always referenced in the antenna frame, is completely specified by two parameters (eg. azimuth and elevation angles) or a line of sight unit vector $\nu=\left[\begin{array}{lll}\nu_{x} & \nu_{y} & \nu_{z}\end{array}\right]^{T}$. Using this definition the array response vector can be written in terms of the components of $\nu$ and a series of vectors $\mathbf{b}_{i}, i=1, \ldots, M$ from the array reference point to the sensor locations.

$$
\mathbf{a}(\theta)=\left[\begin{array}{c}
e^{j \frac{2 \pi}{\lambda} \mathbf{b}_{1}^{T} \nu} \\
e^{j \frac{2 \pi}{\lambda} \mathbf{b}_{2}^{T} \nu} \\
\vdots \\
e^{j \frac{2 \pi}{\lambda} \mathbf{b}_{M}^{T} \nu}
\end{array}\right]
$$

The signal from the $l$ th satellite, $s_{l}(t)$, contains the sampled spreading waveform, and the sampled Doppler referenced at the array reference point, defined as the origin of the antenna frame. The low rate ( $50 \mathrm{~Hz}$ for GPS) data sequence that modulates the fast rate spreading waveform is assumed constant during a collection of snapshots, and is included in the complex gain $\gamma_{l}$.

Equation (6) may now be written in a more compact form as

$$
\mathbf{x}\left(t_{n}\right)=\mathbf{A}(\boldsymbol{\Theta}) \boldsymbol{\Gamma} \mathbf{s}\left(t_{n}\right)+\mathbf{n}\left(t_{n}\right) \quad n=1, \ldots, N
$$

where $\boldsymbol{\Theta}=\left[\theta_{1}, \ldots, \theta_{L}\right], \mathbf{A}(\boldsymbol{\Theta})=\left[\mathbf{a}\left(\theta_{1}\right), \ldots, \mathbf{a}\left(\theta_{L}\right)\right]$, and $\boldsymbol{\Gamma}$ is a diagonal matrix with $\gamma_{1}, \ldots, \gamma_{L}$ along the diagonal. The time varying components of the satellite signals at time $t_{n}$ are contained in $\mathbf{s}\left(t_{n}\right)=\left[s_{1}\left(t_{n}\right), \ldots, s_{L}\left(t_{n}\right)\right]^{T}$.

The model for the interference is left relatively unstructured, allowing for thermal noise, multiple jammers, and other interfering signals. We assume that the interference noise waveform vector, $\mathbf{n}(t)$, is zero mean, wide sense stationary, and circularly symmetric complex Gaussian with positive definite covariance matrix $\mathbf{R}_{s}$, where the subscript " $s$ " implies the spatial, i.e. sensor to sensor covariance. The interference is uncorrelated from all satellite signals. Moreover, we assume the interference is temporally uncorrelated. Since thermal noise will exist in the sensors' data, it is safe to assume that $\mathbf{R}_{s}(0)$ is positive definite.

These assumptions are common for analysis involving jamming or other interfering signals, as in $[5,6]$. A final assumption is that the interference covariance is known. In practice, it is not known, but can be estimated. Methods of estimating the statistics of the interference are presented in $[4,5,7,8]$.

\section{Maximum Likelihood Direction Estimation}

This section briefly reviews maximum likelihood direction finding, to provide a mathematical foundation for the maximum likelihood attitude estimation to follow. Taking the natural logarithm of the likelihood function of the 
received data snapshots $\mathbf{x}\left(t_{n}\right), n=1,2, \ldots, N$ and dropping the constant terms produces the familiar log likelihood ratio (LLR), and the $4 L$ real parameters composing $\hat{\boldsymbol{\Theta}}$ and $\hat{\boldsymbol{\Gamma}}$ that maximize this ratio are the maximum likelihood estimates of $\boldsymbol{\Theta}$ and $\boldsymbol{\Gamma}$ :

$$
\begin{array}{r}
\hat{\boldsymbol{\Theta}}, \hat{\boldsymbol{\Gamma}}=\arg \max _{\Theta, \Gamma} \sum_{n=1}^{N}-\left[\mathbf{x}\left(t_{n}\right)-\mathbf{A}(\boldsymbol{\Theta}) \boldsymbol{\Gamma} \mathbf{y}\left(t_{n}\right)\right]^{H} \mathbf{R}_{s}^{-1} \\
{\left[\mathbf{x}\left(t_{n}\right)-\mathbf{A}(\boldsymbol{\Theta}) \boldsymbol{\Gamma} \mathbf{y}\left(t_{n}\right)\right]}
\end{array}
$$

A significant factor in the estimation of the directions to the satellites (and later, the attitude) is the knowledge of the satellite waveform. Since the waveforms are known, the received data may be de-spread and integrated, increasing the signal to interference ratio. De-modulation of the Doppler and spreading sequence is achieved by projecting the data from each sensor onto the estimated $1 \times N$ temporal steering vector $\hat{\mathbf{y}}_{l}$ for each source. However, since the satellites are assumed in track in both time and frequency, we substitute $\hat{\mathbf{y}}_{l}=\mathbf{y}_{l}$ for $l=1, \ldots, L$, where $\mathbf{y}_{l}$ is defined as:

$$
\mathbf{y}_{l}=\left[s_{l}\left(t_{1}\right), s_{l}\left(t_{2}\right), \ldots, s_{l}\left(t_{N}\right)\right]
$$

Let $\mathbf{u}_{l}$ represent the vector of data demodulated and despread by the $l$ th waveform:

$$
\mathbf{u}_{l}=\frac{1}{\varepsilon_{l}} \sum_{n=1}^{N} \mathbf{x}\left(t_{n}\right) y_{l}^{*}\left(t_{n}\right)
$$

where $\varepsilon_{l}=\mathbf{y}_{l} \mathbf{y}_{l}^{H}=\sum_{n=1}^{N} y_{l}\left(t_{n}\right) y_{l}^{*}\left(t_{n}\right)$.

It can be shown [5] that if the signal waveforms are uncorrelated, as is the case with the GPS satellite waveforms, that the multidimensional estimation problem decomposes to a series of decoupled estimations of individual source parameters. At the stationary point corresponding to the (as yet unknown) estimate of direction $\hat{\theta}_{l}$, the complex gain is found to be:

$$
\hat{\gamma_{l}}=\frac{\mathbf{a}^{H}\left(\hat{\theta}_{l}\right) \mathbf{R}_{s}^{-1} \mathbf{u}_{l}}{\mathbf{a}^{H}\left(\hat{\theta}_{l}\right) \mathbf{R}_{s}^{-1} \mathbf{a}\left(\hat{\theta}_{l}\right)} \quad l=1, \ldots, L
$$

and using this expression provides the estimator for $\theta_{l}$ :

$$
\hat{\theta_{l}}=\arg \max _{\theta_{l}} \frac{\left|\mathbf{a}^{H}\left(\theta_{l}\right) \mathbf{R}_{s}^{-1} \mathbf{u}_{l}\right|^{2}}{\mathbf{a}^{H}\left(\theta_{l}\right) \mathbf{R}_{s}^{-1} \mathbf{a}\left(\theta_{l}\right)} \quad l=1, \ldots, L
$$

\section{A New Array Response Vector}

Direction finding, in loose terms, states that the information provided by each source (satellite in this case), through the differences in time or phase of the source signal as received across the sensors, is a direction. However, for the GPS attitude determination task consider the new concept that each satellite directly provides an (ambiguous) estimate of the antenna attitude when the known direction to the satellite in the local-level frame is included in the estimation process.

We begin by re-examining the array response vector $\mathbf{a}$. Previously this was defined as a function of the two parameter valued $\theta$, which was derived from the line of sight vector $\nu$ (in the antenna frame) to the source, as shown in equation (7). Indeed, for the last 30 years or so of array processing the argument of the array response vector has been the angular direction to the source. However, another equally valid method of parameterizing this expression is in terms of the line of sight vector in the local level frame and the antenna (or body) attitude. These two LOS vectors (using the subscripts B and LL denote body frame and local level frame, respectively) are related by the direction cosine matrix $\mathbf{Q}$ that transforms vectors in local level frame to body frame. The direction cosines that comprise the direction cosine matrix are defined by the body attitude, $q$, as

$$
\nu_{B}=\mathbf{Q}(q) \nu_{L L}
$$

producing the array response vector parameterized on antenna / body attitude:

$$
\mathbf{a}\left(\nu_{L L F}, q\right)=\left[\begin{array}{c}
e^{j \frac{2 \pi}{\lambda} \mathbf{b}_{1}^{T} \mathbf{Q}(q) \nu_{L L}} \\
e^{j \frac{2 \pi}{\lambda} \mathbf{b}_{2}^{T} \mathbf{Q}(q) \nu_{L L}} \\
\vdots \\
e^{j \frac{2 \pi}{\lambda} \mathbf{b}_{M}^{T} \mathbf{Q}(q) \nu_{L L}}
\end{array}\right]
$$

The values of the new array response vectors for each of the local level frame satellite directions evaluated at the true attitude are identical to the values of the standard (direction based) array response vectors evaluated at each of the true satellite directions in antenna frame,

$$
\mathbf{a}\left(\stackrel{o}{\theta_{l}}\right)=\mathbf{a}\left(\nu_{l}, \stackrel{o}{q}\right)
$$

where the $\stackrel{o}{\theta_{l}}$ and $\stackrel{o}{q}$ represent the true direction and attitude.

\section{Attitude Ambiguity}

When the array response vector is parameterized by antenna frame direction, it is typically assumed that the mapping between direction and array response is unique. However, when defined as a function of attitude, the array response vector is ambiguous: an entire family of attitudes will all produce the same array response vector. This ambiguity is the manifestation of the fact that attitude cannot be uniquely resolved when using information from only a single satellite source.

Mathematically, this ambiguity can be obtained from the quaternion representation of attitude. The locus of possible attitudes can be determined by the true attitude quaternion $\stackrel{\mathbf{q}}{\mathbf{q}}$, the local-level frame LOS vector $\nu$ to the satellite source, and a scalar variable $\omega$. Using these, the ambiguity in attitude from the new array response vector definition may be represented as

$$
\mathbf{a}(\stackrel{\mathbf{q}}{\mathbf{q}}, \nu)=\mathbf{a}((\breve{\mathbf{q}}(\omega) \star \stackrel{\mathbf{q}}{\mathbf{q}}), \nu) \quad-\pi \leq \omega<\pi
$$

where the quaternion $\breve{\mathbf{q}}(\omega)$ is

$$
\breve{\mathbf{q}}(\omega)=\left[\begin{array}{c}
\sin \left(\frac{\omega}{2}\right) \nu \\
\cos \left(\frac{\omega}{2}\right)
\end{array}\right]
$$


Theorem 1: The attitude ambiguity in the array response vector for one satellite intersects the ambiguity in the array response vector for a satellite at a different direction in a single attitude, the true attitude of the antenna array. That is,

$$
\mathbf{q}_{1}\left(\omega_{1}\right) \cap \mathbf{q}_{2}\left(\omega_{2}\right)=\stackrel{\mathbf{o}}{\mathbf{q}}
$$

Proof: The proof is straightforward quaternion manipulation and is found in [9].

This theorem is consistent with the conventional GPS attitude determination concept that states the minimum number of satellites required for attitude determination is two [2].

\section{Maximum Likelihood Attitude Estimation}

Since the despreading and Doppler removal is typically performed in GPS receiver hardware as a multiply, integrate, and dump [10], we consider as the observables for this approach the demodulated and integrated data vectors $\mathbf{u}_{l}, l=1,2, \ldots L$.

Recall from equation (11) that $\mathbf{u}_{l}$ contains contributions from all satellites and interference. We denote the signal contribution from the $l$ th satellite as $\mathbf{z}_{l}$ and the interference (including jammers, thermal noise, and the multiple access interference from other satellites) as $\mathbf{w}_{l}$, so that

$$
\mathbf{u}_{l}=\mathbf{z}_{l}+\mathbf{w}_{l}
$$

where

$$
\begin{aligned}
\mathbf{z}_{l} & =\frac{1}{\varepsilon_{l}} \gamma_{l} \mathbf{a}\left(\nu_{l}, \stackrel{o}{q}\right) \mathbf{y}_{l} \hat{\mathbf{y}}_{l}^{H} \\
& =\gamma_{l} \mathbf{a}\left(\nu_{l}, \stackrel{o}{q}\right)
\end{aligned}
$$

and

$$
\begin{aligned}
\mathbf{w}_{l}=\frac{1}{\varepsilon_{l}}\left[\mathbf{n}\left(t_{1}\right), \mathbf{n}\left(t_{2}\right), \ldots,\right. & \left.\mathbf{n}\left(t_{N}\right)\right] \mathbf{y}_{l}^{H} \\
& +\frac{1}{\varepsilon_{l}} \sum_{p=1, p \neq l}^{L} \gamma_{p} \mathbf{a}_{p}(\stackrel{o}{q}) \mathbf{y}_{p} \mathbf{y}_{l}^{H}
\end{aligned}
$$

Just as with the thermal noise and jammer signals, we consider $\mathbf{w}_{l}$ to be a zero mean, circularly symmetric, complex vector of Gaussian ${ }^{2}$ random variables. It is clear that $\mathbf{u}_{l}$ is a consistent estimator of $\mathbf{z}_{l}$.

$$
\begin{aligned}
\mathrm{E}\left[\mathbf{w}_{l}\right] & =0 \\
\mathrm{E}\left[\mathbf{w}_{l} \mathbf{w}_{l}^{T}\right] & =0
\end{aligned}
$$

Using the these, we define the space-satellite data vector, $\mathbf{U}$, of the received data as:

$$
\mathbf{U}=\left[\begin{array}{llll}
\mathbf{u}_{1}^{T} & \mathbf{u}_{2}^{T} & \ldots & \mathbf{u}_{L}^{T}
\end{array}\right]^{T}
$$

$\mathbf{Z}$ and $\mathbf{W}$, the components of $\mathbf{U}$, are similarly defined such that

$$
\mathbf{U}=\mathbf{Z}+\mathbf{W}
$$

${ }^{2}$ Using this model for the multiple access interference is similar to approaches used for bit error analysis of multi-user communication systems.
Finally, let $\mathbf{R}_{s s}$ denote the $M L \times M L$ space-satellite covariance formed from $\mathbf{W}$ as

$$
\mathbf{R}_{s s}=\mathrm{E}\left[\mathbf{W} \mathbf{W}^{H}\right]
$$

By employing the new array response vector, the log likelihood function is now parameterized by the antenna attitude and the complex gains, and not the body frame directions to the satellites.

$$
\mathrm{LLR}=[\mathbf{U}-\mathbf{Z}(\boldsymbol{\Gamma}, q)]^{H} \mathbf{R}_{s s}^{-1}[\mathbf{U}-\mathbf{Z}(\boldsymbol{\Gamma}, q)]
$$

Due to the orthogonal nature of the GPS spreading sequences, it can be shown [9] that the $M L \times M L$ spacesatellite interference covariance matrix asymptotically reduces to a block diagonal structure of $M \times M$ blocks:

$$
\mathbf{R}_{s s}=\left[\begin{array}{cccc}
\mathbf{C}_{1} & 0 & \cdots & 0 \\
0 & \mathbf{C}_{2} & 0 & 0 \\
0 & \cdots & \ddots & 0 \\
0 & \cdots & 0 & \mathbf{C}_{L}
\end{array}\right]
$$

Therefore as $N$ (i.e. the integration time) becomes large, the maximum likelihood estimates of attitude and complex gains asymptotically approach the following sum of terms:

$$
\hat{q}, \hat{\boldsymbol{\Gamma}} \simeq \arg \min _{q, \Gamma} \sum_{l=1}^{L}\left[\mathbf{u}_{l}-\gamma_{l} \mathbf{a}\left(\nu_{l}, q\right)\right]^{H} \mathbf{C}_{l}^{-1}\left[\mathbf{u}_{l}-\gamma_{l} \mathbf{a}\left(\nu_{l}, q\right)\right]
$$

As with the direction finding application, a closed form expression for $\gamma_{l}$ in terms of $q$ at a stationary point is:

$$
\hat{\gamma_{l}}=\frac{\mathbf{a}^{H}\left(\nu_{l}, q\right) \mathbf{C}_{l}^{-1} \mathbf{u}_{l}}{\mathbf{a}^{H}\left(\nu_{l}, q\right) \mathbf{C}_{l}^{-1} \mathbf{a}\left(\nu_{l}, q\right)}
$$

However, unlike the direction finding scenario, the estimator for attitude is coupled, that is all satellites (being tracked) contribute to the attitude estimate. Substituting (31) into (30) and removing terms that do not vary with attitude produces the following attitude estimator:

$$
\hat{q}=\arg \max _{q} \sum_{l=1}^{L} \frac{\left.\mid \mathbf{a}\left(\nu_{l}, q\right)\right]\left.^{H} \mathbf{C}_{l}^{-1} \mathbf{u}_{l}\right|^{2}}{\mathbf{a}^{H}\left(\nu_{l}, q\right) \mathbf{C}_{l}^{-1} \mathbf{a}\left(\nu_{l}, q\right)}
$$

By using a shortened nomenclature, a "whitened" array response vector may be defined as:

$$
\tilde{\mathbf{a}}_{l}(q)=\mathbf{C}_{l}^{-1 / 2} \mathbf{a}\left(\nu_{l}, q\right)
$$

where $\mathbf{C}_{l}=\mathbf{C}_{l}^{1 / 2} \mathbf{C}_{l}^{1 / 2}$ Equation (32) may now be rewritten as

$$
\begin{aligned}
\hat{q} & =\max _{q} \sum_{l=1}^{L} \frac{\left|\mathbf{u}_{l}^{H} \mathbf{C}_{l}^{-1 / 2} \tilde{\mathbf{a}}_{l}(q)\right|^{2}}{\left|\tilde{\mathbf{a}}_{l}(q)\right|^{2}} \\
& =\max _{q} \sum_{l=1}^{L} \mathbf{u}_{l}^{H} \mathbf{C}_{l}^{-1 / 2} \mathbf{P}_{\tilde{\mathbf{a}}_{l}(q)} \mathbf{C}_{l}^{-1 / 2} \mathbf{u}_{l}
\end{aligned}
$$

where

$$
\mathbf{P}_{\tilde{\mathbf{a}}_{l}(q)}=\frac{\tilde{\mathbf{a}}_{l}(q) \tilde{\mathbf{a}}_{l}^{H}(q)}{\tilde{\mathbf{a}}_{l}^{H}(q) \tilde{\mathbf{a}}_{l}(q)}
$$


Equation (35) is the Maximum Likelihood Attitude Estimator (MLAE). One way to view this expression is that it produces a "metric" value at every possible attitude, and the attitude producing the lowest metric value is chosen as the estimate. Since each satellite source contributes to the metric, the attitude ambiguity is removed and the complementary geometric structure of the satellite constellation is utilized to provide a high quality estimate of attitude.

\section{MLAE PROPERTIES}

Even though both the MLAE and the multi-source direction estimator of [5] make use of known and uncorrelated source waveforms, they are fundamentally different, since the direction estimates obtained from the algorithm of [5] are independent source to source while the MLAE attitude estimate is formed from information coupled across all satellites. However, we can show that the MLAE, like the direction estimators, is asymptotically consistent, and efficient.

Consider the right side of equation (35) as the function $G(q)$ of the unknown attitude:

$$
G(q)=\sum_{l=1}^{L} \mathbf{u}_{l}^{H} \mathbf{C}_{l}^{-1 / 2} \mathbf{P}_{\tilde{\mathbf{a}}_{l}(q)} \mathbf{C}_{l}^{-1 / 2} \mathbf{u}_{l}
$$

$G(q)$ is simply the value of the estimator "metric," parameterized on the continuum of hypothesized attitudes of the GPS antenna array. We also define the following vectors and matrices:

$$
\begin{aligned}
\mathbf{d}_{i}(l) & =\frac{\partial \mathbf{a}_{l}(q)}{\partial q_{i}} \\
\tilde{\mathbf{d}}_{i}(l) & =\frac{\partial \tilde{\mathbf{a}}_{l}(q)}{\partial q_{i}} \\
\mathbf{D}(l) & =\left[\mathbf{d}_{1}(l) \mathbf{d}_{2}(l) \mathbf{d}_{3}(l)\right] \\
\tilde{\mathbf{D}}(l) & =\left[\tilde{\mathbf{d}}_{1}(l) \tilde{\mathbf{d}}_{2}(l) \tilde{\mathbf{d}}_{3}(l)\right] \\
\tilde{\mathbf{a}}_{l}^{\dagger}(q) & =\left(\tilde{\mathbf{a}}_{l}^{H}(q) \tilde{\mathbf{a}}_{l}(q)\right)^{-1} \tilde{\mathbf{a}}_{l}^{H}(q) \\
\tilde{\mathbf{w}}_{i}(l) & =\mathbf{C}_{l}^{-1 / 2} \mathbf{w}_{i}(l)
\end{aligned}
$$

The subscript $(i)$ in the derivative vectors $\mathbf{d}_{i}(l)$ above indicates the attitude parameter with respect to which the derivative is being taken, while the value in parentheses $(l)$ indicates the satellite to which the array response vector corresponds.

Theorem 2: The MLAE is a consistent ${ }^{3}$ estimator for $^{-}$ antenna attitude. That is,

$$
\lim _{N \rightarrow \infty} \hat{q}=\stackrel{o}{q}
$$

Proof: Consider the contribution of only the first satellite to $G(q)$ in equation (37), and define this contribution as $G_{1}(q)$ :

$$
G_{1}(q)=\mathbf{u}_{1}^{H} \mathbf{C}_{1}^{-1 / 2} \mathbf{P}_{\tilde{\mathbf{a}}_{1}(a)} \mathbf{C}_{1}^{-1 / 2} \mathbf{u}_{1}
$$

${ }^{3} \mathrm{~A}$ consistent estimator has the desirable property that the estimation error decreases as the number of data samples increases.
Recall that $\mathbf{u}_{l}$ is a consistent estimator of $\gamma_{l} \mathbf{a}\left(\nu_{1}, \stackrel{o}{q}\right)$. Then asymptotically, (45) will converge to the maximum of

$$
\left|\gamma_{1}\right|^{2} \frac{\left|\tilde{\mathbf{a}}\left(\nu_{1}, q\right)^{H} \tilde{\mathbf{a}}\left(\nu_{1}, \stackrel{o}{q}\right)\right|^{2}}{\tilde{\mathbf{a}}\left(\nu_{1}, q\right)^{H} \tilde{\mathbf{a}}\left(\nu_{1}, q\right)}
$$

where $\tilde{\mathbf{a}}\left(\nu_{1}, q\right)$ is defined in (33) One could look at equation (46) and ascertain, by the Cauchy-Schwarz inequality, that the attitude that maximizes $G_{1}$ is $\stackrel{o}{q}$. However, this would not be entirely correct. Indeed, $\stackrel{o}{q}$ would be one attitude to maximize $G_{1}$. But since the array response vector is ambiguous when parameterized in attitude, an entire family of attitudes will maximize $G_{1}$. Let the quaternion $\mathbf{q}_{1}\left(\omega_{1}\right)$ represent the family of attitudes that asymptotically maximize (46). Then using (17) and (18), $\mathbf{q}_{1}\left(\omega_{i}\right)$ can be written as

$$
\mathbf{q}_{1}\left(\omega_{1}\right)=\breve{\mathbf{q}}_{1}\left(\omega_{1}\right) \star \stackrel{\mathbf{q}}{\mathbf{q}}
$$

where $\star$ represents quaternion multiplication.

Now consider the remaining satellites' contributions. In a similar fashion as with satellite one, the family of attitudes $\mathbf{q}_{2}\left(\omega_{2}\right)$ that asymptotically minimize the second through $L$ th satellite's contribution to (37) can be found as

$$
\mathbf{q}_{i}\left(\omega_{i}\right)=\breve{\mathbf{q}}_{i}\left(\omega_{i}\right) \star \mathbf{q} \quad i=2, \ldots, L
$$

For the $L$ satellites, there now exist $L$ families of attitudes that separately minimize each satellites contribution to (37). In general, the parameter value that minimizes a sum of functions is not necessarily the value that minimizes any particular function. However, from Theorem 1, each of these families of minima intersect in a single point, the true attitude $\stackrel{\mathbf{q}}{\mathbf{q}}$. So asymptotically, the estimated attitude is the true attitude, and therefore the MLAE is consistent.

Now consider the function $G(q)$ of equation (37) near the estimated attitude $\hat{q}$, and the Taylor series expansion of the gradient vector $\mathbf{g}^{\prime}(q)$ near $\hat{q}[11]$. The gradient is a $3 \times 1$ vector, since there are three independent attitude parameters. Since $\hat{q}$ maximizes $G(q)$, the gradient $\mathbf{g}^{\prime}(\hat{q})$ at this stationary point is zero. If the difference between the estimate and true value is small ${ }^{4}$, then the higher order terms in the Taylor series expansion may be ignored, leaving:

$$
0=\mathbf{g}^{\prime}(\hat{q})=\mathbf{g}^{\prime}(\stackrel{o}{q})+\mathbf{g}^{\prime \prime}(\stackrel{o}{q})(\hat{q}-\stackrel{o}{q})+\cdots
$$

such that

$$
(\hat{q}-\stackrel{o}{q})=-\left[\mathbf{g}^{\prime \prime}(\stackrel{o}{q})\right]^{-1} \mathbf{g}^{\prime}(\stackrel{o}{q})
$$

where

$$
\mathbf{g}^{\prime}(q) \triangleq\left[\begin{array}{lll}
\frac{\partial G(q)}{\partial q_{1}} & \frac{\partial G(q)}{\partial q_{2}} & \frac{\partial G(q)}{\partial q_{3}}
\end{array}\right]^{T}
$$

and

$$
\mathbf{g}^{\prime \prime}(q) \triangleq\left(\begin{array}{lll}
\frac{\partial^{2} G(q)}{\partial q_{1} \partial q_{1}} & \frac{\partial^{2} G(q)}{\partial q_{1} \partial q_{2}} & \frac{\partial^{2} G(q)}{\partial q_{1} \partial q_{3}} \\
\frac{\partial^{2} G(q)}{\partial q_{2} \partial q_{1}} & \frac{\partial^{2} G(q)}{\partial q_{2} \partial q_{2}} & \frac{\partial^{2} G(q)}{\partial q_{2} \partial q_{3}} \\
\frac{\partial^{2} G(q)}{\partial q_{3} \partial q_{2}} & \frac{\partial^{2} G(q)}{\partial q_{3} \partial q_{2}} & \frac{\partial^{2} G(q)}{\partial q_{3} \partial q_{3}}
\end{array}\right)
$$


and $q_{1}, q_{2}$, and $q_{3}$ are the three attitude parameters. By straightforward evaluation, we find four key relationships needed to evaluate equation (50):

$$
\begin{array}{r}
\mathbf{g}^{\prime}(q) \simeq 2 \operatorname{Re}\left\{\sum_{\mathrm{l}=1}^{\mathrm{L}} \gamma_{1}^{*} \tilde{\mathbf{D}}^{\mathrm{H}}(\mathrm{l}) \mathbf{P}_{\tilde{\mathbf{a}}_{1}(\stackrel{\mathrm{q}}{\mathrm{q}})}^{\perp} \tilde{\mathbf{w}}_{\mathrm{l}}\right\} \\
\mathbf{g}^{\prime \prime}(q)=-2 \operatorname{Re}\left\{\sum_{\mathrm{l}=1}^{\mathrm{L}}\left|\gamma_{1}\right|^{2} \tilde{\mathbf{D}}^{\mathrm{H}}(\mathrm{l}) \mathbf{P}_{\tilde{\mathbf{a}}_{\mathrm{l}}}^{\perp} \tilde{\mathbf{D}}(\mathrm{l})\right\} \\
\mathrm{E}\left[\mathbf{g}^{\prime}(\stackrel{o}{q})\left(\mathbf{g}^{\prime}(\stackrel{o}{q})\right)^{T}\right] \simeq \\
2 \operatorname{Re}\left\{\sum_{\mathrm{l}=1}^{\mathrm{L}}\left|\gamma_{1}\right|^{2} \tilde{\mathbf{D}}^{\mathrm{H}}(\mathrm{l}) \mathbf{P}_{\tilde{\mathbf{a}}_{\mathrm{l}}}^{\perp} \tilde{\mathbf{D}}(\mathrm{l})\right\} \\
\mathrm{E}\left[\mathbf{g}^{\prime}(\stackrel{o}{q})\right] \simeq 0
\end{array}
$$

From the last relationship above it is clear that the MLAE is asymptotically unbiased.

Theorem 3: The asymptotic covariance matrix of $\hat{q}$, the attitude estimated with the MLAE, is given by

$\mathrm{E}\left[(\hat{\mathrm{q}}-\stackrel{\mathrm{o}}{\mathrm{q}})(\hat{\mathrm{q}}-\stackrel{\mathrm{o}}{\mathrm{q}})^{\mathrm{T}}\right] \simeq\left[2 \operatorname{Re}\left\{\sum_{\mathrm{l}=1}^{\mathrm{L}}\left|\gamma_{1}\right|^{2} \tilde{\mathbf{D}}^{\mathrm{H}}(\mathrm{l}) \mathbf{P}_{\tilde{\mathbf{a}}_{1}}^{\perp} \tilde{\mathbf{D}}(\mathrm{l})\right\}\right]^{-1}$

Proof: The estimation error covariance matrix may be evaluated using (50):

$$
\begin{aligned}
& \mathrm{E}\left[(\hat{\mathrm{q}}-\stackrel{\mathrm{o}}{\mathrm{q}})(\hat{\mathrm{q}}-\stackrel{\mathrm{o}}{\mathrm{q}})^{\mathrm{T}}\right] \simeq \\
& \quad \mathrm{E}\left[\left[\mathbf{g}^{\prime \prime}(\stackrel{\mathrm{o}}{\mathrm{q}})\right]^{-1} \mathbf{g}^{\prime}(\stackrel{\mathrm{o}}{\mathrm{q}})\left(\mathbf{g}^{\prime}(\stackrel{\mathrm{o}}{\mathrm{q}})\right)^{\mathrm{T}}\left[\mathbf{g}^{\prime \prime}(\stackrel{\mathrm{o}}{\mathrm{q}})\right]^{-1}\right]
\end{aligned}
$$

We may replace the terms involving $\mathbf{g}^{\prime \prime}(\stackrel{o}{q})$ by their (deterministic) asymptotic values, represented as $\mathbf{g}_{a}^{\prime \prime}(\stackrel{o}{q})=$ $-2 \operatorname{Re}\left\{\sum_{\mathrm{l}=1}^{\mathrm{L}}\left|\gamma_{\mathrm{l}}\right|^{2} \tilde{\mathbf{D}}^{\mathrm{H}}(\mathrm{l}) \mathbf{P}_{\tilde{\mathbf{a}}_{\mathrm{l}}}^{\perp} \tilde{\mathbf{D}}(\mathrm{l})\right\}:$

$$
\begin{aligned}
& \mathrm{E}\left[(\hat{\mathrm{q}}-\stackrel{\mathrm{o}}{\mathrm{q}})(\hat{\mathrm{q}}-\stackrel{\mathrm{o}}{\mathrm{q}})^{\mathrm{T}}\right] \simeq \\
& \quad\left[\mathbf{g}_{a}^{\prime \prime}(\stackrel{\circ}{\mathrm{q}})\right]^{-1} \mathrm{E}\left[\mathbf{g}^{\prime}(\stackrel{\mathrm{o}}{\mathrm{q}})\left(\mathbf{g}^{\prime}(\stackrel{\mathrm{o}}{\mathrm{q}})\right)^{\mathrm{T}}\right]\left[\mathbf{g}_{\mathrm{a}}^{\prime \prime}(\stackrel{\mathrm{o}}{\mathrm{q}})\right]^{-1}
\end{aligned}
$$

Using (55) to address the quantity involved in the expectation, and observing that that quantity is the negative inverse of $\mathbf{g}_{a}^{\prime \prime}(\stackrel{o}{q})$ completes the proof.

Theorem 4: The Cramér-Rao Bound on attitude estimates, when the interference covariance is known and each satellite return is scaled by an unknown complex gain, is given by the compact expression

$$
P_{C R}(q)=\left[2 \operatorname{Re}\left\{\sum_{\mathrm{l}=1}^{\mathrm{L}}\left|\gamma_{1}\right|^{2} \tilde{\mathbf{D}}^{\mathrm{H}}(\mathrm{l}) \mathbf{P}_{\tilde{\mathbf{a}}(\mathrm{l})}^{\perp} \tilde{\mathbf{D}}(\mathrm{l})\right\}\right]^{-1}
$$

Proof: See the Appendix.

Since $P_{q} \simeq P_{C R}(q)$, the MLAE is asymptotically an efficient estimator of antenna attitude.

\section{SIMULATION RESULTS}

In this section simulation results are used to illustrate performance differences between conventional attitude estimation and the new MLAE in a jammed environment. Three antenna topologies are used for the studies below. The first antenna uses 4 elements on a rectangularly sampled grid, while the second and third use 4 and 7 elements on a triangularly sampled grid in a "Y" and hexagonal shape, respectively. Figure 2 shows the antenna locations in wavelengths for these antennas. Each of these is chosen with the maximum inter-element spacing allowed that prevents grating lobes in visible space ${ }^{5}$ [12].

The relevant parameters of the simulated receiver are as follows. The receiver chain simulated has a system noise figure of $4 \mathrm{~dB}$, a coherent signal loss of $5 \mathrm{~dB}$, and a noncoherent signal loss of $2 \mathrm{~dB}$. Each receiver hardware channel is assumed identical. The satellite power received at the antenna is $-163 \mathrm{~dB}$. The jammer Effective Radiated Power (ERP) is 20 watts, and the jammers are located 20 nautical miles from the antenna. Multipath, either from the jammer or satellites, is not simulated for the studies below. As is assumed throughout this work, all satellites used for attitude determination are assumed to be in code and Doppler track.

Each realization of the simulation produces an attitude estimate using the estimators discussed in the previous chapters. There are several ways in which the simulation results may be presented, but the one employed in this work, is to calculate the total error for each realization. The total error is the angular rotation required to rotate the antenna from the estimated attitude to the correct attitude, and is always greater than or equal to zero. For each scenario, the mean total error across all realizations in that scenario is calculated for each of the estimation methods as the average of the total errors from each simulation realization.

Figures 3 and 4 present the performance of the conventional attitude estimation approach and the new MLAE as a function of update rate (inverse of integration time). Both algorithms were evaluated using the three antenna topologies described above. In Figure 3, a single jammer with North-East-Down LOS direction vector $[.45, .6,-.66]$ is visible, while in Figure 4 three jammers at directions $[.45, .6,-.66],[.7,0,-.71]$, and $[.45,-.6,-.66]$ exist. As expected, the conventional approach provides unacceptable performance even with only a single jammer visible and at long integration times. However, the MLAE error is significantly smaller than the conventional approach throughout the range of update rates and number of jammers simulated.

\section{CONCLUSIONS}

This work has developed an interference resistant algorithm for GPS based attitude determination, the MLAE. The MLAE optimally includes information from all satellites in view, and its performance is shown herein to asymptotically achieve the Cramér-Rao Bound (CRB),

\footnotetext{
${ }^{5}$ This is to simulate a GPS system that uses an antenna array to provide anti-jam for position location.
} 


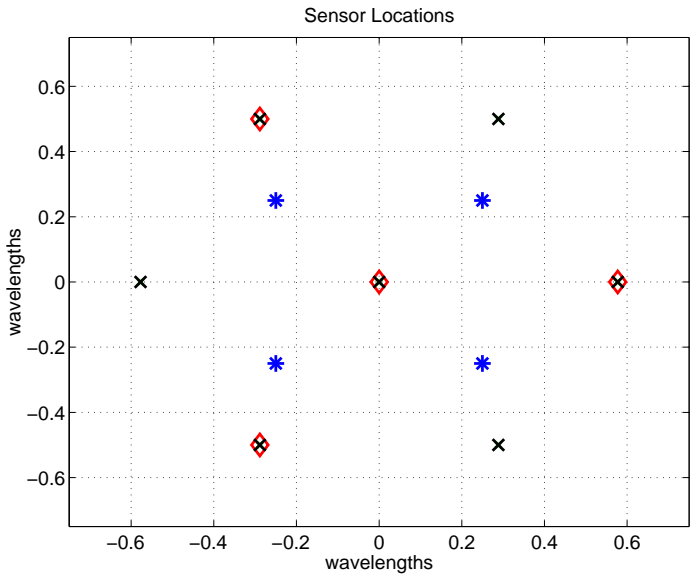

Fig. 2. Sensor locations for the three antenna topologies. Quad (asterisk), Y (diamond) and $\operatorname{Hex}(\mathrm{x})$.

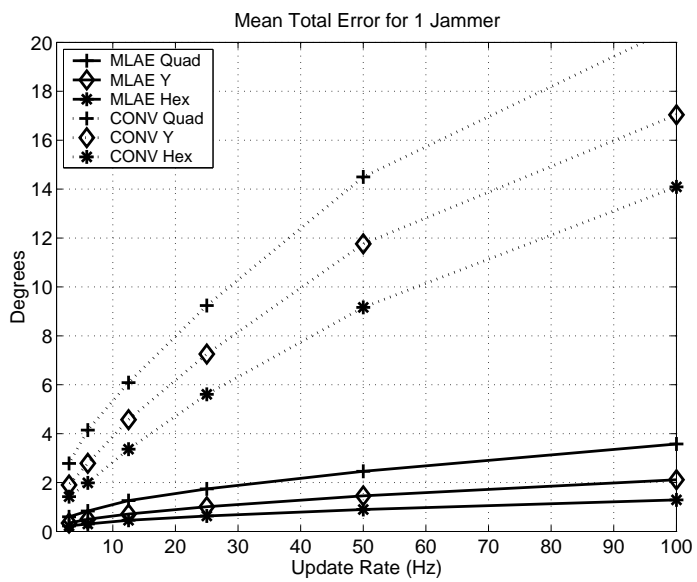

Fig. 3. Conventional and MLAE Mean Total Error Performance in a one jammer scenario.

i.e. the MLAE asymptotically achieves the performance limit for unbiased estimators. Simulation results demonstrate that this approach provides a significant increase in performance over conventional attitude determination methods in a strong interference environment.

\section{APPENDIX}

\section{Cramér-Rao Bound}

The Cramér-Rao Bound (CRB) provides a lower bound on the mean squared error (MSE) of the estimation error for an unbiased estimator. An estimator whose MSE achieves the CRB is said to be statistically efficient [13].

The Cramér-Rao Bound (CRB) matrix, $\mathbf{P}_{c r}$, is

$$
\mathbf{P}_{c r}=\left(\mathrm{E}\left\{\left[\frac{\partial \ln f_{\chi}(\chi \mid \rho)}{\partial \rho}\right]\left[\frac{\partial \ln f_{\chi}(\chi \mid \rho)}{\partial \rho}\right]^{T}\right\}\right)^{-1}
$$

where $\rho$ is a real vector of the unknown parameters, and $f_{\chi}(\chi \mid \rho)$ is the likelihood function. Before performing the matrix inversion, the right side of the equation (i.e. $\mathbf{P}_{c r}^{-1}$ ) is the "Fisher Information Matrix," FIM. For attitude determination, $\rho$ consists of the three unknown parameters of attitude ${ }^{6}, q_{i}, i=1,2,3$ and the $2 \times L$ real components

\footnotetext{
${ }^{6} \mathrm{~A}$ convenient parameterization of the three attitude parameters
}

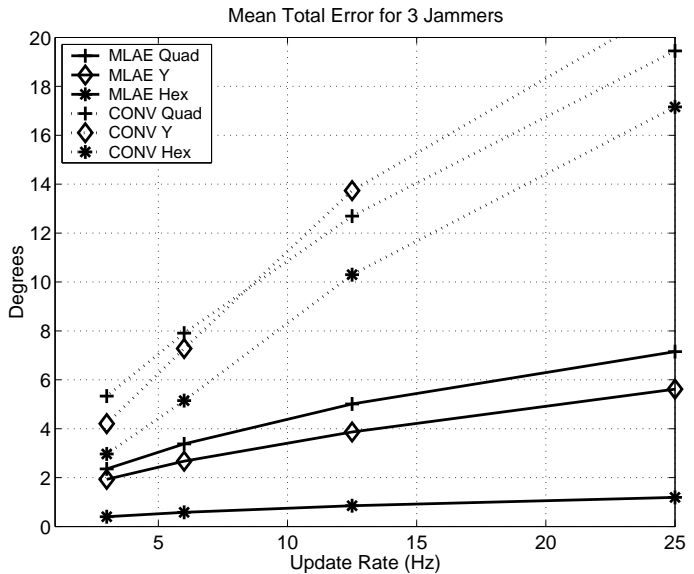

Fig. 4. Conventional and MLAE Mean Total Error Performance in a three jammer scenario.

of the $L$ complex gains, in the following order.

$$
\begin{aligned}
\rho=\left[\begin{array}{cccccc}
q_{1} & q_{2} & q_{3} & \operatorname{Re}\left(\gamma_{1}\right) & \operatorname{Re}\left(\gamma_{2}\right) & \ldots \\
\operatorname{Re}\left(\gamma_{\mathrm{L}}\right) & \operatorname{Im}\left(\gamma_{1}\right) & \operatorname{Im}\left(\gamma_{2}\right) & \ldots & \operatorname{Im}\left(\gamma_{\mathrm{L}}\right)
\end{array}\right]
\end{aligned}
$$

The natural logarithm of the likelihood equation is

$$
\begin{array}{r}
\ln f_{\chi}(\chi \mid \rho)=-\sum_{l=1}^{L}\left[\mathbf{u}_{l}-\gamma_{l} \mathbf{a}_{l}(q)\right]{ }^{H} \mathbf{C}_{l}^{-1}\left[\mathbf{u}_{l}-\gamma_{l} \mathbf{a}_{l}(q)\right] \\
+\{\text { constant terms }\}
\end{array}
$$

In order to evaluate (61) (i.e. to generate the various terms of the FIM), the following three derivative formulas are required:

$$
\begin{aligned}
& \frac{\partial \ln f_{\chi}(\chi \mid \rho)}{\partial \operatorname{Re}\left(\gamma_{1}\right)}=2 \operatorname{Re}\left\{\mathbf{a}_{1}^{\mathrm{H}}(\mathrm{q}) \mathbf{C}_{\mathbf{l}}^{-\mathbf{1}} \mathbf{w}_{\mathbf{l}}\right\} \\
& \frac{\partial \ln f_{\chi}(\chi \mid \rho)}{\partial \operatorname{Im}\left(\gamma_{1}\right)}=2 \operatorname{Im}\left\{\mathbf{a}_{1}^{\mathrm{H}}(\mathrm{q}) \mathbf{C}_{\mathbf{l}}^{-\mathbf{1}} \mathbf{w}_{\mathbf{l}}\right\}
\end{aligned}
$$

$$
\frac{\partial \ln f_{\chi}(\chi \mid \rho)}{\partial q_{i}}=2 \operatorname{Re}\left\{\sum_{\mathrm{l}=1}^{\mathrm{L}} \gamma_{1}^{*} \mathbf{d}_{\mathrm{i}}^{\mathrm{H}}(\mathrm{l}) \mathbf{C}_{\mathbf{l}}^{-\mathbf{1}} \mathbf{w}_{\mathbf{l}}\right\}
$$

where $\mathbf{w}_{l}$ is defined in (20) and $\mathbf{d}_{k}(l)$ is defined in equation (38). Derivation of these formulas is straightforward using the results of [11] and is found in [9].

The order of the terms in the vector $\rho$ determines the structure of the FIM. Using equations (61) and (64-66) the FIM may be written as

$$
\mathrm{FIM}=\left[\begin{array}{ccc}
\mathbf{F}_{q} & \operatorname{Re}\left[\mathbf{F}_{\mathrm{q}, \gamma}\right] & \operatorname{Im}\left[\mathbf{F}_{\mathrm{q}, \gamma}\right] \\
\operatorname{Re}\left[\mathbf{F}_{\mathrm{q}, \gamma}\right]^{\mathrm{T}} & \mathbf{F}_{\gamma} & \mathbf{0} \\
\operatorname{Im}\left[\mathbf{F}_{\mathrm{q}, \gamma}\right]^{\mathrm{T}} & \mathbf{0} & \mathbf{F}_{\gamma}
\end{array}\right]
$$

where

for the CRB calculation is to choose the three Euler angles. 


$$
\begin{aligned}
& \mathbf{F}_{\mathbf{q}}= 2 \operatorname{Re}\left\{\sum_{l=1}^{\mathrm{L}}\left|\gamma_{1}\right|^{2} \mathbf{D}^{\mathrm{H}}(\mathrm{l}) \mathbf{C}_{\mathbf{l}}^{-\mathbf{1}} \mathbf{D}(\mathbf{l})\right\} \\
&= 2 \sum_{l=1}^{L}\left|\gamma_{l}\right|^{2} \tilde{\mathbf{D}}^{H}(l) \tilde{\mathbf{D}}(l) \\
& \mathbf{F}_{q, \gamma} 2\left[\begin{array}{l}
\operatorname{diag}\left\{\Gamma \mathbf{A}^{H} \mathbf{C}_{\mathbf{1}}^{-\mathbf{1}} \triangle_{\mathbf{1}}\right\} \\
\operatorname{diag}\left\{\Gamma \mathbf{A}^{H} \mathbf{C}_{\mathbf{1}}^{-\mathbf{1}} \triangle_{\mathbf{2}}\right\} \\
\operatorname{diag}\left\{\Gamma \mathbf{A}^{H} \mathbf{C}_{\mathbf{1}}^{-\mathbf{1}} \triangle_{\mathbf{3}}\right\}
\end{array}\right] \\
&=2 {\left[\begin{array}{l}
\operatorname{diag}\left\{\Gamma \tilde{\mathbf{A}}^{\mathbf{H}} \tilde{\triangle}_{\mathbf{1}}\right\} \\
\operatorname{diag}\left\{\Gamma \tilde{\mathbf{A}}^{\mathbf{H}} \tilde{\triangle}_{\mathbf{2}}\right\} \\
\operatorname{diag}\left\{\tilde{\mathbf{A}}^{\mathbf{H}} \tilde{\triangle}_{\mathbf{3}}\right\}
\end{array}\right] } \\
& \mathbf{F}_{\gamma}=\mathbf{A}^{H} \mathbf{C}_{\mathbf{l}}^{-\mathbf{1}} \mathbf{A} \odot \mathbf{I}_{L} \\
&=\tilde{\mathbf{A}}^{H} \tilde{\mathbf{A}} \odot \mathbf{I}_{L}
\end{aligned}
$$

and $\mathbf{A}$ is a matrix of array response vectors:

$$
\begin{aligned}
& \mathbf{A}=\left[\begin{array}{llll}
\mathbf{a}_{1}(q) & \mathbf{a}_{2}(q) & \cdots & \mathbf{a}_{L}(q)
\end{array}\right] \\
& \tilde{\mathbf{A}}=\left[\begin{array}{llll}
\tilde{\mathbf{a}}_{1}(q) & \tilde{\mathbf{a}}_{2}(q) & \cdots & \tilde{\mathbf{a}}_{L}(q)
\end{array}\right]
\end{aligned}
$$

and the $\triangle_{i}$ and $\tilde{\triangle}_{i}$ are $M \times L$ matrices of array response derivatives

$$
\begin{aligned}
\triangle_{i} & =\left[\begin{array}{llll}
\mathbf{d}_{i}(1) & \mathbf{d}_{i}(2) & \cdots & \mathbf{d}_{i}(L)
\end{array}\right] \\
\tilde{\triangle}_{i} & =\left[\begin{array}{llll}
\tilde{\mathbf{d}}_{i}(1) & \tilde{\mathbf{d}}_{i}(2) & \cdots & \tilde{\mathbf{d}}_{i}(L)
\end{array}\right]
\end{aligned}
$$

and $\operatorname{diag}(x)$ forms a row or column of the diagonal elements of the square matrix $\mathbf{x}$ as appropriate. ${ }^{7}$

Of interest in this problem is the CRB for the attitude; the complex gain terms are essentially nuisance parameters. The CRB for the desired attitude parameters, $\mathbf{P}_{c r}(q)$, is found from the upper $3 \times 3$ block of the inverse of the FIM. Using the inverse identity of [5],

$$
\mathbf{P}_{c r}(q)^{-1}=\operatorname{Re}\left\{\mathbf{F}_{\mathrm{q}}-\mathbf{F}_{\mathrm{q}, \gamma} \mathbf{F}_{\gamma}^{-1} \mathbf{F}_{\mathrm{q}, \gamma}^{\mathrm{H}}\right\}
$$

Now examine the terms of $\mathbf{P}_{c r}^{-1}(q)$. Since $\mathbf{F}_{\gamma}$ is a diagonal matrix, $\left(\mathbf{F}_{\gamma}\right)^{-1}$ is also diagonal. Using this, the $i, j$ element of $\mathbf{F}_{q, \gamma} \mathbf{F}_{\gamma}^{-1} \mathbf{F}_{q, \gamma}^{H}$ is found to be

$$
\begin{array}{r}
\mathbf{F}_{q, \gamma} \mathbf{F}_{\gamma}^{-1} \mathbf{F}_{q, \gamma}^{H}(i, j)=2 \sum_{l=1}^{L}\left|\gamma_{l}\right|^{2} \mathbf{d}_{i}^{H}(l) \mathbf{C}_{\mathbf{l}}^{-\mathbf{1}} \mathbf{a}(\mathbf{l}) * \\
\left(\frac{1}{\mathbf{a}^{H}(l) \mathbf{C}_{\mathbf{l}}^{-\mathbf{1}} \mathbf{a}(\mathbf{l})}\right) \mathbf{a}^{H}(l) \mathbf{C}_{\mathbf{l}}^{-\mathbf{1}} \mathbf{d}_{\mathbf{j}}(\mathbf{l})
\end{array}
$$

${ }^{7}$ For $n \times n$ matrix $\mathbf{x}$, this operation is,

$$
\operatorname{diag}(\mathbf{x})=\left[\begin{array}{llll}
1 & 1 & \cdots & 1
\end{array}\right]\left(\mathbf{x} \odot \mathbf{I}_{n}\right)
$$

which can be written as

$$
\mathbf{F}_{q, \gamma} \mathbf{F}_{\gamma}^{-1} \mathbf{F}_{q, \gamma}^{H}(i, j)=2 \sum_{l=1}^{L}\left|\gamma_{l}\right|^{2} \tilde{\mathbf{d}}_{i}^{H}(l) \mathbf{P}_{\tilde{\mathbf{a}}(l)} \tilde{\mathbf{d}}_{j}(l)
$$

The entire $3 \times 3$ matrix of $\mathbf{F}_{q, \gamma} \mathbf{F}_{\gamma}^{-1} \mathbf{F}_{q, \gamma}^{H}$ is

$$
\mathbf{F}_{q, \gamma} \mathbf{F}_{\gamma}^{-1} \mathbf{F}_{q, \gamma}^{H}=2 \sum_{l=1}^{L}\left|\gamma_{l}\right|^{2} \tilde{\mathbf{D}}_{i}^{H}(l) \mathbf{P}_{\tilde{\mathbf{a}}(l)} \tilde{\mathbf{D}}(l)
$$

Finally, recall that $\mathbf{F}_{q}=2 \operatorname{Re}\left\{\sum_{\mathrm{l}=1}^{\mathrm{L}}\left|\gamma_{\mathrm{l}}\right|^{2} \tilde{\mathbf{D}}^{\mathrm{H}}(\mathrm{l}) \tilde{\mathbf{D}}(\mathrm{l})\right\}$, so that

$$
\mathbf{P}_{c r}^{-1}(q)=2 \operatorname{Re}\left\{\sum_{\mathrm{l}=1}^{\mathrm{L}}\left|\gamma_{1}\right|^{2} \tilde{\mathbf{D}}^{\mathrm{H}}(\mathrm{l}) \mathbf{P}_{\tilde{\mathbf{a}}(\mathrm{l})}^{\perp} \tilde{\mathbf{D}}(\mathrm{l})\right\}
$$

which concludes the proof.

\section{REFERENCES}

[1] Christopher Comp, GPS Carrier Phase Multipath Characterization and a Mitigation Technique Using the Signal-to-Noise Ratio, Ph.D. dissertation, Department of Aerospace Engineering Sciences, University of Colorado, 1996.

[2] Clark E. Cohen, "Attitude determination," in Global Positioning System: Theory and Applications, Bradford W. Parkinson and James J. Spilker JR., Eds., vol. 2, pp. 519-538. American Institude of Aeronautics and Astronautics, INC., 1996.

[3] Frank Van Graas and Michael Braasch, "GPS interferometric attitude and heading determination: Initial flight test results," Navigation: Journal of the Institute of Navigation, vol. 38, no. 4, pp. 297-316, Winter 1991-1992.

[4] Matthew Markel, Eric Sutton, and Henry Zmuda, "An antenna array-based approach to attitude determination in a jammed environment," Proceedings of the ION GPS '01, September 2001.

[5] Jian Li, Bijit Halder, Petre Stoica, and Mats Viberg, "Computationally efficient angle estimation for signals with known waveforms," IEEE Transactions on Signal Processing, vol. 43, no. 9, pp. 2154-2163, September 1995.

[6] Jian Li and R. T. Compton, "Maximum likelihood angle estimation for signals with known waveforms," IEEE Transactions on Signal Processing, vol. 41, no. 9, pp. 2850-2862, September 1993.

[7] Mark A. Jones and Mark. A. Wickert, "Direct-sequence spreadspectrum using directionally constrained adaptive beam forming to null interference," IEEE Journal on Selected Areas in Communications, vol. 13, no. 1, pp. 71-79, January 1995.

[8] Chandra Vaidyanathan, Kevin M. Buckley, and Srinath Hosur, "A blind adaptive antenna array for CDMA systems," ASILOMAR Conference on Signals, Systems, and Computers, pp. 1373-1377, 1995.

[9] Matthew Markel, Interference Mitigation for GPS Based Attitude Determination, Ph.D. dissertation, University of Florida, May 2002.

[10] Elliot D. Kaplan, Understanding GPS Principles and Applications, Artech House, 1996.

[11] Petre Stoica and Ayre Nehorai, "MUSIC, Maximum Likelihood, and Cramer-Rao Bound," IEEE Transactions on Acoustics, Speech, and Signal Processing, vol. 37, no. 5, pp. 720-741, May 1989.

[12] Richard C. Johnson, Antenna Engineering Handbook, chapter 20, McGraw-Hill, 1993.

[13] Petre Stoica and Randolph Moses, Introduction to Spectral Analysis, Prentice Hall, 1997.

where $\mathbf{I}_{n}$ is an $n \times n$ identity matrix. 